\title{
A sensorless method for measuring the point mobility of mechanical structures
}

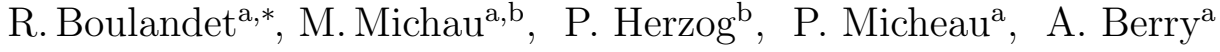 \\ ${ }^{a}$ Groupe d'Acoustique de l'Université de Sherbrooke, Université de Sherbrooke, J1K 2R1, \\ Sherbrooke, Canada \\ ${ }^{b}$ Laboratoire de Mécanique et d'Acoustique, UPR CNRS 7051, 13453, Marseille cedex 13, \\ France
}

\begin{abstract}
This paper presents a convenient and cost-effective experimental tool for measuring the mobility characteristics of a mechanical structure. The objective is to demonstrate that the point mobility measurement can be performed using only an electrodynamic inertial exciter. Unlike previous work based on voice coil actuators, no load cell or accelerometer is needed. Instead, it is theoretically shown that the mobility characteristics of the structure can be estimated from variations in the electrical input impedance of the actuator fixed onto it, provided that the electromechanical parameters of the actuator are known. The proof of concept is made experimentally using a cheap commercially available actuator on a simply supported plate, leading to a good dynamic range from $100 \mathrm{~Hz}$ to $1 \mathrm{kHz}$. The methodology to assess the basic parameters of the actuator is also given. Measured data are compared to a standard shaker testing and the strengths and weaknesses of the sensorless mobility measuring device are discussed. It is believed that this sensorless
\end{abstract}

\footnotetext{
*Corresponding author

Email address: romain.boulandet@gmail.com (R. Boulandet)
}

Preprint submitted to Journal of Sound and Vibration

May 13, 2016

CC 2016. This manuscript version is made available under the Elsevier user license http://www.elsevier.com/open-access/userlicense/1.0/ 
mobility measuring device can be a convenient experimental tool to determine the dynamic characteristics of a wide range of mechanical structures.

\section{Introduction}

The concepts of mechanical impedance and mobility are part of the design process and analysis in structural dynamics. Developed from electromechanical and electroacoustic analogies in the 1920's [1, 2, 3, 4], they define the relationship between force and velocity at given locations of a structure. The point mobility characteristics of a mechanical structure, which is based on a collocated measurement of force and velocity, has broad and practical applications in: 1) modal testing to determine the natural frequencies, modal damping and mode shape of structures [5], 2) materials testing to determine certain specific properties of materials such as the loss factor and the modulus of elasticity [6], 3) structural monitoring in order to investigate non-uniformities, structural defects or tolerance deviation that can be detected by comparing the mobility function of a standard with a recently manufactured item [7]. It is also used in system modelling since mobility measurement performed at many locations on a structure can be used as verification of mathematical models, or the response of subsystems or cascaded substructures may be measured and the properties of the assembled system be predicted before final assembly [8], similarly to the study of complex electrical circuits. In smart structures and vibration isolation, furthermore, it allows the dynamic interaction between electromechanical transducers and structure to be involved in the development process [9]; vibration isolator, proof-mass actuator and machinery mounting can then be optimized to minimize structural vibration 
or sound transmission [11, 12].

The measurement of mechanical impedance and mobility in general requires three devices : an actuator, a force sensor and a vibration sensor [6, 8, 13]. In impact testing, the structure under test is excited by a sharp and clean force input of small time duration, generally by using an impact hammer equipped with a load cell, and the vibration response is typically sensed by using an accelerometer. In shaker testing, a vibrating-rod actuator is required to mechanically excite the structure according to the amplified input signal. In both cases mechanical energy is supplied to the structure with a known frequency content, allowing the mobility function to be calculated from the force applied and the vibration response. Achieving collocated sensing is a challenging task and imperfect colocation may result in significant errors in the measured mobility function [14]. An alternative approach for the direct measurement of the driving point mobility of a structure has recently been proposed by Sands et al. [15] (2009). In this method, an electrodynamic inertial actuator combined with two accelerometers is attached to the structure directly so that the assembly can be used as a mobility measuring device. The two sensors are actually placed strategically on the actuator frame and proof mass, allowing the mass-loading effect of the device on the structure to be removed. The feasibility of the method was validated in [15] on a cantilever beam. Although this approach overcomes some practical limitations encountered in impact testing and shaker testing, such as allowing better control over the frequency, amplitude and duration of the force excitation applied to the structure while compensating the mass-loading effect of the actuator on the structure, it requires developing a specific device. On the other hand, 

2 both sensor and actuator simultaneously, a technique which is commonly 3 referred to as self-sensing actuation in the literature. The idea of self-sensing 4 actuation was developed in the early nineties by Hagood et al. [16] and Dosch 5

several authors have studied how to use electromechanical transducers as actuation was developed in the early nineties by Hagood et al. [16 and Dosch et al. [17] concurrently, where the piezoceramic elements were used as sensors and actuators simultaneously to reduce implementation, cost and complexity, by achieving truly collocated control. The principle was later implemented to achieve active control of noise and vibration [18, 19, 20, 21], to implement self-sensing vibration damping using electrodynamic shaker actuators [22], for active acoustic attenuation [23] and for biomedical applications using an electromagnetic actuator [24]. More recently, the principle has been applied to an electrodynamic inertial exciter for active vibration control of a simply supported plate [9] and for active structural acoustic control of an aircraft panel [10]. In particular, it was shown in Ref. [10] that the vibration of the structure at the actuator base can be estimated from the actuator impedances and the current and voltage at the transducer's terminals, paving the way for the development of a sensorless mobility measuring device. The approach of estimating the mechanical impedance and mobility of a structure from the electrical impedance of an electromechanical transducer attached onto it is not new. In the mid 1990s, studies on the coupling of the electrical impedance of piezoceramic materials and the mechanical impedance of structure at the location of the piezo patch were reported by Liang et al. [25]. In the early 2000s, Ling et al. developed a technique for measuring the mechanical impedance of structures using the sensing capability of a piezoceramic inertial actuator [26]. Experimental results for monitoring structural integrity of a 

4 a methodology for determining the loss factor and the Young's modulus of

clamped-clamped beam can be found in [27], showing that some structural defects can be detected by examining the changes of the electrical impedance of the actuator. In the footsteps of Ref. [26], Doutres et al. (2010) developed a porous sample from the measurement of the electrical impedance of an electrodynamic loudspeaker used to apply static and dynamic stress on it [28]. Bois et al. (2007) also proposed a method for detecting and characterizing a delamination process in a composite structure using embedded piezoelectric transducers, which allows to determine the modal parameters from electromechanical impedance measurements [29].

This paper intends to simplify the mobility measuring device proposed by Sands et al. [15] by jointly using the self-sensing capabilities of the inertial exciter, as suggested in Ref. [10]. The proposed sensorless mobility measuring device (SMMD) therefore enables the dynamic properties of mechanical structures to be evaluated quickly and inexpensively. The remainder of the paper is organized as follows. Section 2 provides a dynamic model of the electrodynamic actuator as a two-port network, including its transduction functions that are used in the analytical derivation of the mobility function of the structure. In Section 3, we present the methodology used to experimentally evaluate the electromechanical parameters of the actuator needed to determine the transduction functions of the actuator. The proof of concept is provided in Section 4 on the basis of a simply supported plate and experimental results are compared to standard shaker testing. The strengths and weaknesses of the SMMD are discussed in Section 5, including the possible reasons for the observed limitations on the current prototype. 


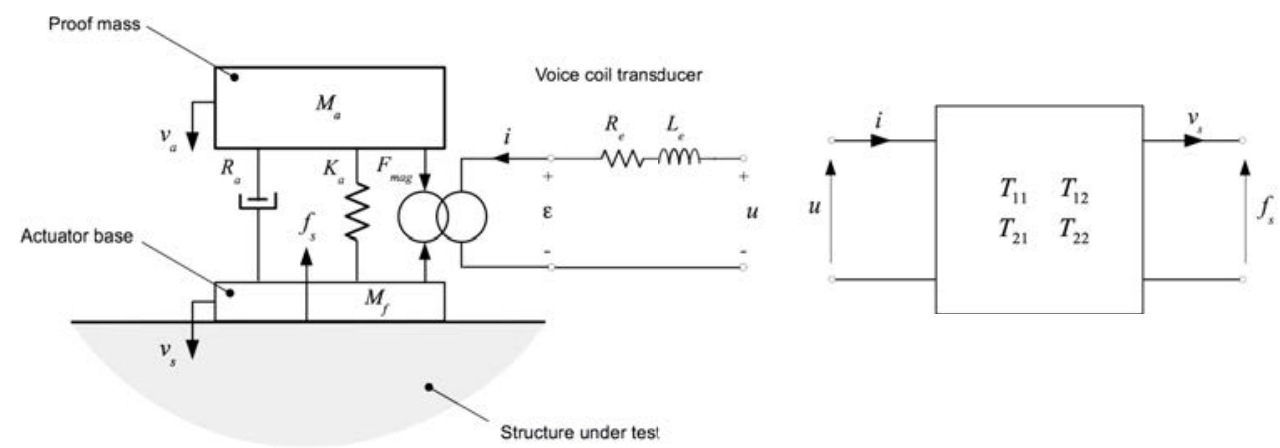

(a)

(b)

Figure 1: Schematic of the electrodynamic inertial exciter (a) and representation as a two-port network (b), where the current $i$ is defined positive when flowing into the electrical port of the transducer and the two collinear velocities $v_{a}$ and $v_{s}$ are defined positive when oriented towards the structure; $u$ is the voltage at the input terminals and $f_{s}$ the reaction force exerted by the structure at the actuator base.

\section{The electrodynamic sensoriactuator}

This section provides the theoretical basis about the operation and char3 acteristics of the electrodynamic inertial exciter, and explains how it can be 4 employed for self-sensing actuation. A lumped parameter model is used in

5 the following assuming $\exp (\mathrm{j} \omega t)$ harmonic dependence and linear behavior. 6 Otherwise, some of the model parameters would need to be considered as 7 time-varying nonlinear functions of the input variables [30].

\subsection{Governing equations}

9 Figure 1 gives a schematic representation of the actuator. The equations of motion of the proof mass $M_{a}$ and frame mass $M_{f}$, and the governing equation 
1 of the electrical circuit can be expressed as [10, 12, 32,

$$
\begin{aligned}
j \omega M_{a} v_{a} & =Z_{m s}(\omega)\left(v_{s}-v_{a}\right)-B l i \\
\mathrm{j} \omega M_{f} v_{s} & =-Z_{m s}(\omega)\left(v_{s}-v_{a}\right)+B l i-f_{s} \\
u & =Z_{e}(\omega) i+B l\left(v_{s}-v_{a}\right)
\end{aligned}
$$

2 where $Z_{m s}(\omega)=R_{a}+K_{a} / \mathrm{j} \omega$ is the mechanical impedance of the spring3 dashpot mounting system and $Z_{e}(\omega)=R_{e}+\mathrm{j} \omega L_{e}$ is the blocked electrical 4 impedance of the transducer.

5

6

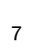

\subsection{Transduction matrix}

The electrodynamic inertial exciter is a linear power device that can be modelled as a two-port network. As illustrated in Fig. 11(b), the complex magnitudes of effort (voltage) and flow (current) at the input (electrical) port are related to the complex magnitudes of effort (force) $f_{s}$ and flow (velocity) $v_{s}$ at the output (mechanical) port, and the governing equations can be represented in transduction matrix by [26, 31]

$$
\left[\begin{array}{l}
u \\
i
\end{array}\right]=\left[\begin{array}{cc}
T_{11}(\omega) & T_{12}(\omega) \\
T_{21}(\omega) & T_{22}(\omega)
\end{array}\right]\left[\begin{array}{l}
f_{s} \\
v_{s}
\end{array}\right]
$$

where the frequency dependent parameters $T_{11}(\omega)=\left.\frac{u}{f_{s}}\right|_{v_{s}=0}$ and $T_{21}(\omega)=$ $\left.\frac{i}{f_{s}}\right|_{v_{s}=0}$ are the transduction function of voltage to force and the transduction function of current to force, respectively, when the actuator base is fixed to a rigid foundation; $T_{12}(\omega)=\left.\frac{u}{v_{s}}\right|_{f_{s}=0}$ and $T_{22}(\omega)=\left.\frac{i}{v_{s}}\right|_{f_{s}=0}$ are the transduction function of voltage to velocity and the transduction function of current to velocity, respectively, when the actuator base is free to vibrate. 
The transduction functions $T_{11}(\omega), T_{21}(\omega), T_{12}(\omega)$ and $T_{22}(\omega)$ given in Eq. (2) can be derived as

$$
\begin{aligned}
& T_{11}(\omega)=\left.\frac{u}{f_{s}}\right|_{v_{s}=0}=\frac{1}{\mathrm{j} \omega M_{a} B l}\left(Z_{e}(\omega) Z_{m a}(\omega)+(B l)^{2}\right) \\
& T_{21}(\omega)=\left.\frac{i}{f_{s}}\right|_{v_{s}=0}=\frac{Z_{m a}(\omega)}{\mathrm{j} \omega M_{a} B l} \\
& T_{12}(\omega)=\left.\frac{u}{v_{s}}\right|_{f_{s}=0}=\frac{Z_{e}(\omega)}{B l}\left(\frac{M_{f}}{M_{a}} Z_{m a}(\omega)+Z_{m s}(\omega)\right)+B l\left(1+\frac{M_{f}}{M_{a}}\right) \\
& T_{22}(\omega)=\left.\frac{i}{v_{s}}\right|_{f_{s}=0}=\frac{1}{B l}\left(\frac{M_{f}}{M_{a}} Z_{m a}(\omega)+Z_{m s}(\omega)\right)
\end{aligned}
$$

where $Z_{m a}(\omega)=Z_{m s}(\omega)+\mathrm{j} \omega M_{a}$, and the determinant of the transduction 2 matrix yields

$$
T=T_{11}(\omega) T_{22}(\omega)-T_{12}(\omega) T_{21}(\omega)=-1
$$

3

4 tromechanical transducer. Fig. 2 shows the block diagram and the analogous

5

6 bility function (in $\mathrm{m} \mathrm{s}^{-1} \mathrm{~N}^{-1}$ ) of the structure to which it is attached is defined 7 as

$$
Y(\omega)=\frac{v_{s}}{f_{s}}
$$

\subsection{The sensoriactuator transfer functions}

9

of the actuator can be derived from Eqs. (3) as

$$
Z_{i n 0}(\omega)=\left.\frac{u}{i}\right|_{v_{s}=0}=\frac{T_{11}(\omega)}{T_{21}(\omega)}
$$




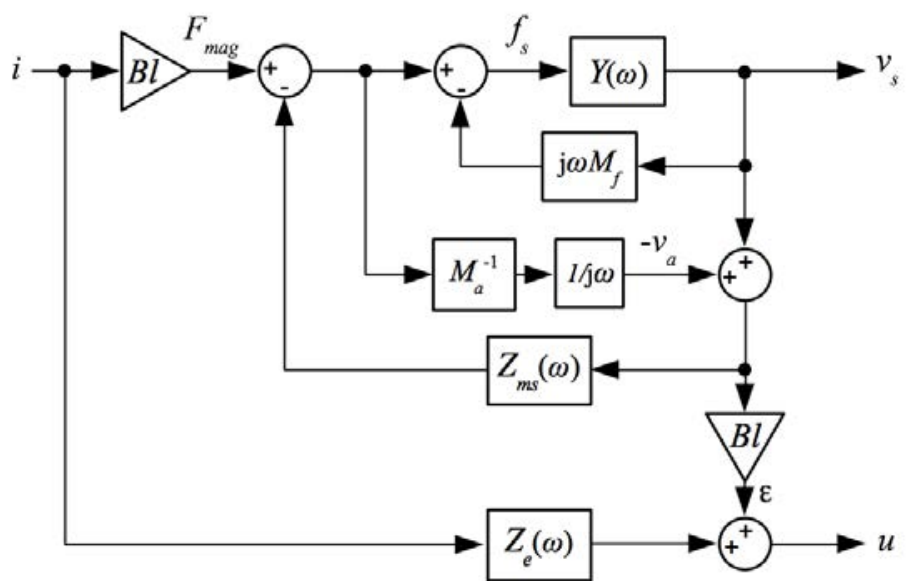

(a)

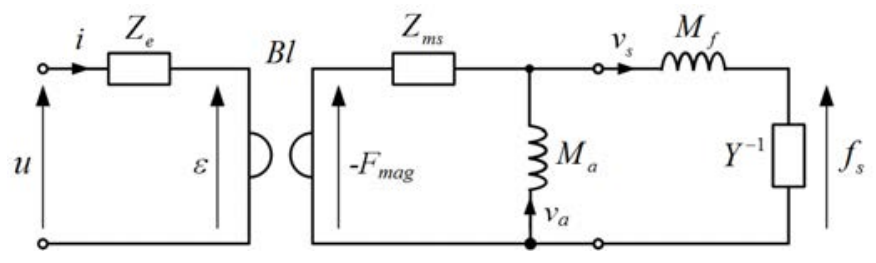

(b)

Figure 2: Block diagram (a) and analogous electrical circuit representation (b) of the current-driven electrodynamic inertial actuator when attached to a flexible structure, derived from Eqs. (1) and (8).

1 Substituting Eq. (8) into Eq. (2), the transfer function of the reaction 2 force to the input current (in $\mathrm{N} \mathrm{A}^{-1}$ ) can be written as

$$
H_{i}(\omega)=\frac{f_{s}}{i}=\left[T_{21}(\omega)+T_{22}(\omega) Y(\omega)\right]^{-1}
$$

3 and the electrical input impedance of the actuator when attached to the 4 structure can be derived as

$$
Z_{i n}(\omega)=\frac{u}{i}=\frac{T_{11}(\omega)+T_{12}(\omega) Y(\omega)}{T_{21}(\omega)+T_{22}(\omega) Y(\omega)}
$$


1

4 current as

$$
G_{i}(\omega)=\frac{v_{s}}{i}=T_{21}(\omega)\left(\frac{T_{11}(\omega)+T_{12}(\omega) Y(\omega)}{T_{21}(\omega)+T_{22}(\omega) Y(\omega)}-\frac{T_{11}(\omega)}{T_{21}(\omega)}\right)=T_{21}(\omega)\left(Z_{i n}(\omega)-Z_{i n 0}(\omega)\right)
$$

5 As can be seen in Eq. 12, the open loop response function $\left(\right.$ in $\mathrm{m} \mathrm{s}^{-1} \mathrm{~A}^{-1}$ ) of 6 the actuator at the attachment point can be readily obtained from variations in 7 the electrical input impedance of the actuator. More details on the analytical 8 derivation of Eqs. 10-12) are given in Appendix A.

\subsection{The sensorless mobility measuring device}

From Eqs. (8-12), and after some further manipulations, the mobility function of the structure at the attachment point can be expressed as

$$
Y(\omega)=\frac{T_{21}(\omega) G_{i}(\omega)}{1-T_{22}(\omega) G_{i}(\omega)}
$$

Therefore, the proposed method to use the electrodynamic inertial exciter as a sensorless mobility measuring device requires 1) deriving the transduction functions $T_{21}$ and $T_{22}$ given in Eqs. (4) and (6), respectively, by evaluating the electromechanical parameters $M_{a}, K_{a}, R_{a}, B l, M_{f}$, and 2) obtaining the open loop response function of the actuator as given in Eq. 12 by measuring the electrical input impedance of the actuator when attached to a rigid foundation and to the structure under test, as indicated in Eqs. (9) and (11), respectively. 


\section{Experimental assessment of electromechanical parameters}

2 This section presents the method employed to evaluate of the basic pa3 rameters of the electrodynamic actuator described in Section 2 based on a 4 transmissibility test and electrical impedance measurement.

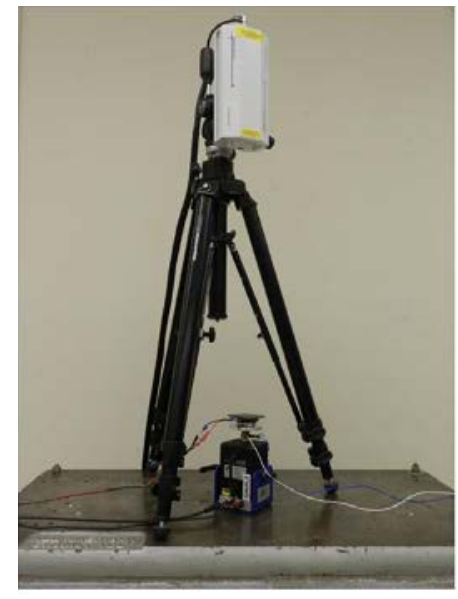

(a)

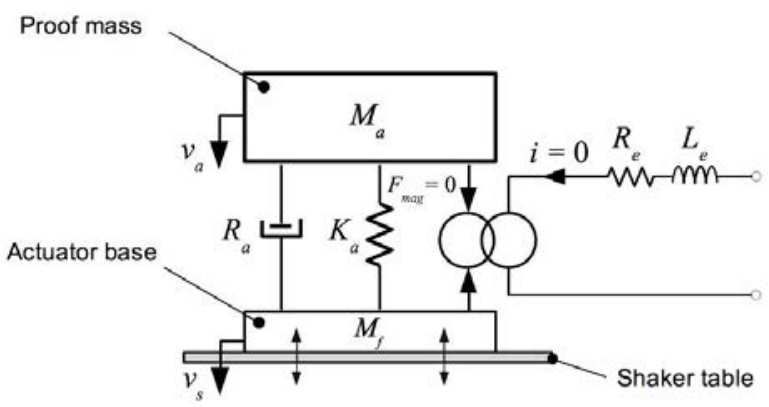

(b)

Figure 3: Picture (a) and schematic representation (b) of the experimental setup used to assess the velocity transmissibility of the actuator when attached to a shaker table.

5 3.1. Experimental setup

$6 \quad$ Figure 3 shows the experimental setup used to assess the velocity transmis7 sibility of the actuator. As shown in Fig. 3, a K2007E01 Mini SmartShaker 8 (5 lbf rms) with integrated power amplifier is used to mechanically drive the 9 inertial exciter and a VibroMet 500V laser Doppler velocimeter (sensitivity $\left.{ }_{10} 5 \mathrm{mV} /\left(\mathrm{m} \mathrm{s}^{-1}\right)\right)$ is used to sense the axial velocity $v_{a}$ of the proof mass $M_{a}$. 11 A PCB model 288D01 impedance head (sensitivity $22.4 \mathrm{mV} / \mathrm{N}$ for the force 


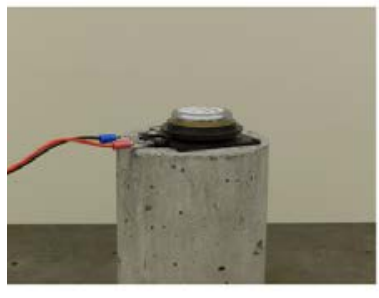

(a)

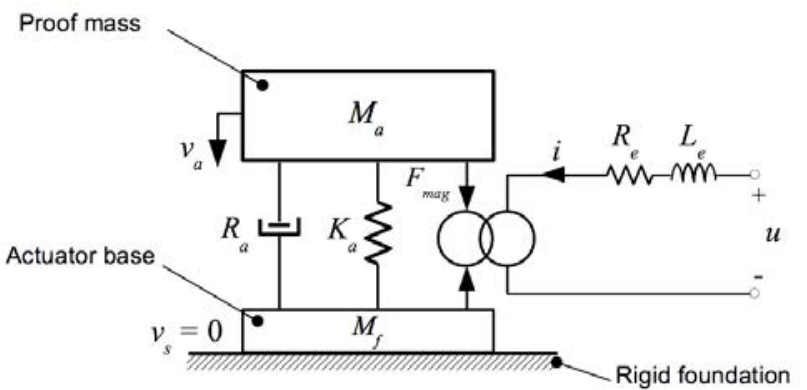

(b)

Figure 4: Picture (a) and schematic representation (b) of the experimental setup used to measure the electrical motional impedance of the actuator when attached to a rigid foundation.

1 sensor and $10.3 \mathrm{mV} /\left(\mathrm{m} \mathrm{s}^{-2}\right)$ for the accelerometer $)$ is connected between the 2 shaker table and the mounting system to which the actuator is attached, 3 allowing the force and acceleration transmitted by the shaker table to be 4 monitored. The accelerometer signal is processed by a time integrator, thus 5 determining the axial velocity $v_{s}$ at the base of the inertial exciter.

$6 \quad$ The electrical input impedance is determined by applying a sweep signal 7 to the current driven actuator while monitoring the voltage induced at its 8 terminals. As shown in Fig. 4, measurement is performed when the actua9 tor base is attached to a rigid foundation, allowing the electrical motional impedance to be evaluated as given in Eq. (9). Similar to the procedure for the determination of electromechanical parameters of a loudspeaker driver [35], measurement is performed at small input level around the resonance frequency of the proof mass on its suspension so that the mechanical behavior of the 14 drive unit is effectively linear. 


\subsection{Measured data}
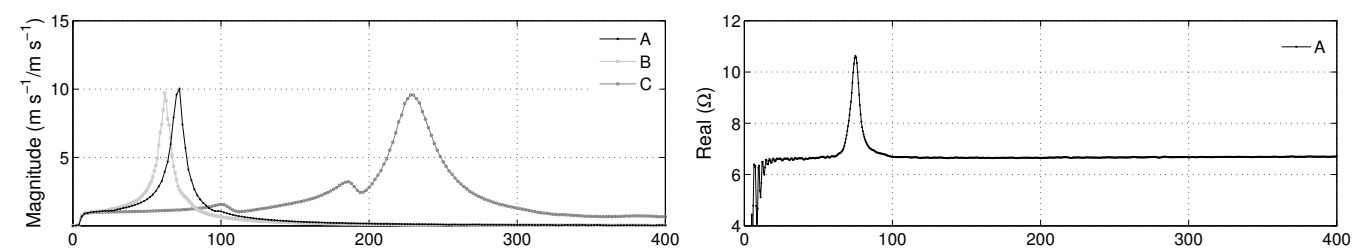

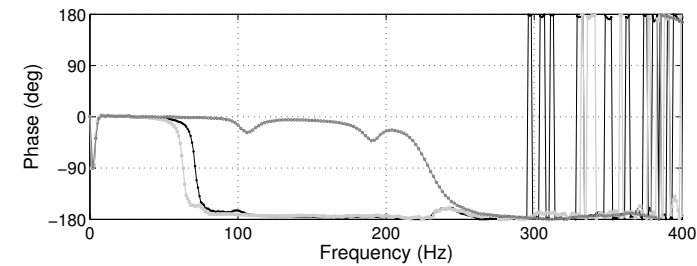

(a)

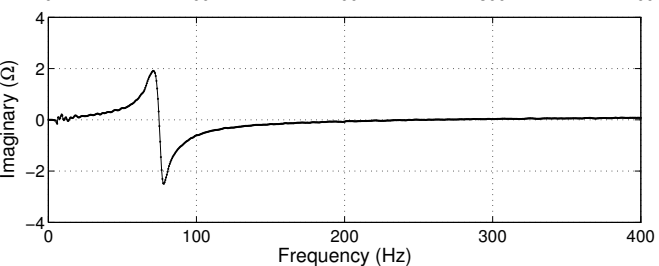

(b)

Figure 5: Frequency response function of the velocity transmissibility (a) measured when the actuator is attached to the shaker table and electrical motional impedance (b) measured when the actuator is attached to a rigid foundation (A: actuator attached at its base, B: attached at its base with the added mass, C: mounted upside down on the shaker).

Figure 5 shows the measured frequency response functions (FRFs) of the 3 velocity transmissibility and electrical motional impedance of the actuator. ${ }_{4}$ As can be seen in Fig. 5, the actuator resonance frequency measured at ${ }_{5} f_{n}=75 \mathrm{~Hz}$ (case A) is found to be shifted at $f_{m}=62 \mathrm{~Hz}$ with an added mass 6 $m=0.0305 \mathrm{~kg}$ (case B). As shown in Appendix B, this measurement is used to 7 determine the proof mass and effective stiffness of the actuator $M_{a}=0.108 \mathrm{~kg}$ 8 and $K_{a} \simeq 24000 \mathrm{Nm}^{-1}$, respectively. When mounted upside down on 9 the shaker table as shown in Fig. 6(c), the actuator resonance frequency is found to be shifted at $f_{f}=222 \mathrm{~Hz}$, thus giving the mass of the actuator 11 base $M_{f}=0.0134 \mathrm{~kg}$. As shown in Fig. 5(a), the peak-transmissibility 
1 equals 9.56 for all configurations tested, thus giving the effective mechanical 2 resistance of the actuator $R_{a}=5.32 \mathrm{~N} \mathrm{~s} \mathrm{~m}^{-1}$. As can be seen in Fig. 5(b), 3 the dc resistance of the coil is $R_{e}=6.65 \Omega$ and the maximum electrical 4 resistance at the actuator resonance frequency is $R_{e m}=10.63 \Omega$, thus giving 5 the electrodynamic transduction coefficient $B l=4.6 \mathrm{~N} \mathrm{~A}^{-1}$. The measured 6 parameters of the actuator used in this study are summarized in Tab.1. More 7 details about the derivation of the basic parameters of the actuator can be 8 found in Appendix B.

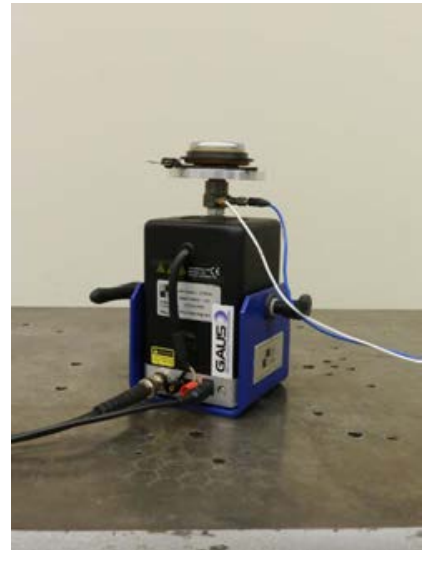

(a)

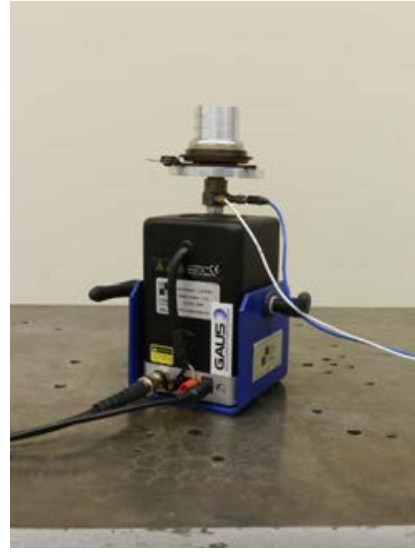

(b)

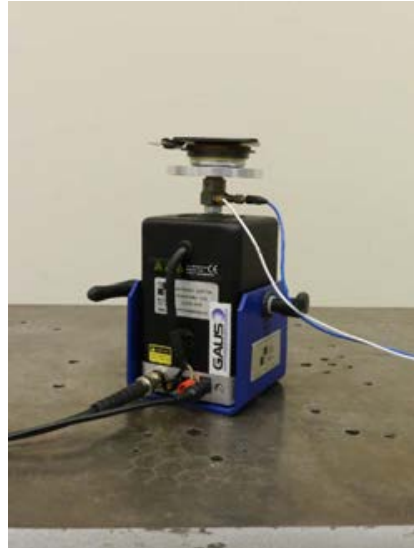

(c)

Figure 6: Pictures of the actuator when attached to the shaker table at its base without (a) and with the added mass (b), and mounted upside down on the shaker table (c).

\section{Experimental proof of concept}

This section aims to experimentally confirm the use of an electrodynamic 
1 mobility characteristics of a structure without the need for an external sensor.

2 For the purpose of illustration, the results presented below relate specifically

3 to a simply supported plate.

4 4.1. Experimental setup

5 The plate used for the tests is shown in Fig. 7, and its physical parameters

6 are summarized in Table 2. More details on the dynamic model of the simply

7 supported plate can be found in [36]. First, a Brüel and Kjaer Type 4810

8 electrodynamic mini-shaker (2.25 lbf pk) is employed to produce the input

9 force, as can be seen in Fig. 7(a). A PCB model 208B04 force sensor, the

10 sensitivity of which is $1.174 \mathrm{mV} / \mathrm{N}$, is connected between the plate and the

Table 1: Physical parameters of the VISATON EX 60 S electrodynamic exciter measured in small-signal range.

\begin{tabular}{lccc}
\hline Parameter & Notation & Value & Unit \\
\hline Coil resistance & $R_{e}$ & 6.65 & $\Omega$ \\
Coil inductance & $L_{e}$ & 0.05 & $\mathrm{mH}$ \\
Transduction coefficient & $B l$ & 4.6 & $\mathrm{~N} \mathrm{~A}^{-1}$ \\
Proof mass & $M_{a}$ & 0.108 & $\mathrm{~kg}$ \\
Frame mass & $M_{f}$ & 0.0134 & $\mathrm{~kg}$ \\
Mechanical resistance & $R_{a}$ & 5.32 & $\mathrm{~N} \mathrm{~s} \mathrm{~m}^{-1}$ \\
Suspension stiffness & $K_{a}$ & $24 \times 10^{3}$ & $\mathrm{~N} \mathrm{~m}^{-1}$ \\
Resonance frequency & $f_{n}$ & 75 & $\mathrm{~Hz}$ \\
\hline
\end{tabular}




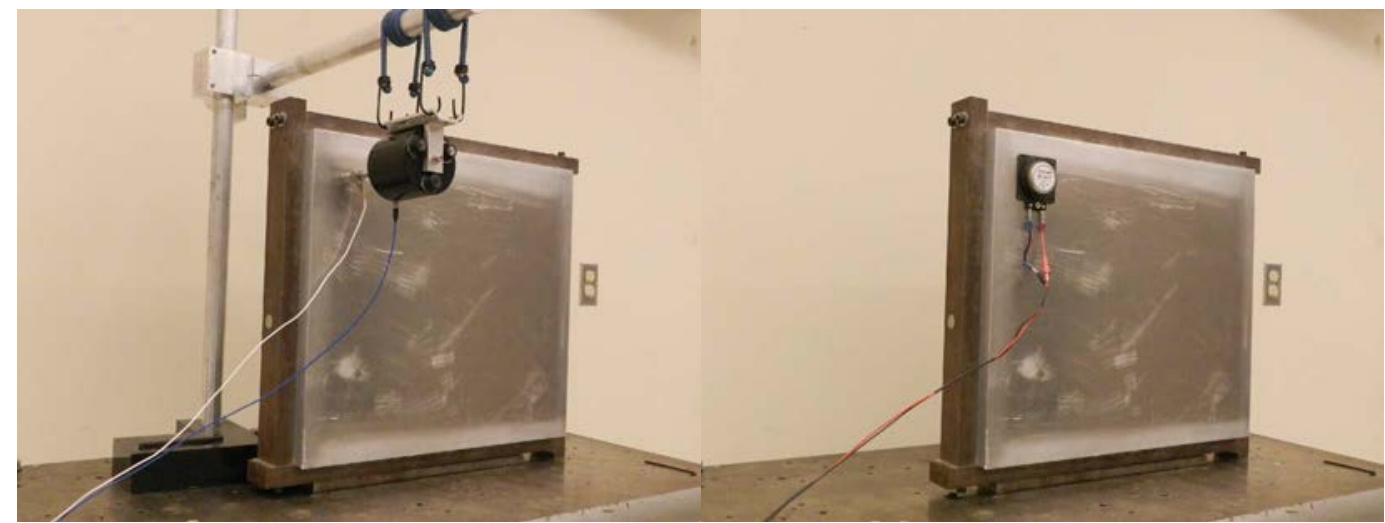

(a)

(b)

Figure 7: Pictures of the simply supported plate equipped for shaker testing (a) and with the sensorless mobility measuring device (b).

1 shaker using a stinger. As illustrated in Fig. 7(b), the SMMD is then fixed 2 near the same location in order to be compared to the measurement obtained 3 with the shaker. Unlike the shaker, the inertial actuator will exert a reaction 4 force on the structure which is due to acceleration of the proof mass, without 5 the need of a grounded support. Not shown in Fig. 7, a VibroMet 500V 6 laser Doppler velocimeter (sensitivity $5 \mathrm{mV} /\left(\mathrm{m} \mathrm{s}^{-1}\right)$ ) is used here to sense 7 the vibration response on the back side of the plate. Signal acquisition is 8 done with 18 bit precision analog inputs at a sampling frequency of $20 \mathrm{kHz}$. 9 The digital signal processing is performed using a Speedgoat $\mathbb{R}$ Performance 11 of the VISATON EX $60 \mathrm{~S}$ electrodynamic exciter used in this study can 12 be found in Tab. 1. A picture of the inertial actuator used to perform the 13 experiments is given in Fig. 8(a). 
Table 2: Physical parameters of the rectangular aluminum plate.

\begin{tabular}{lccc}
\hline Parameter & Notation & Value & Unit \\
\hline Width & $L_{x}$ & 0.481 & $\mathrm{~m}$ \\
Length & $L_{y}$ & 0.421 & $\mathrm{~m}$ \\
Thickness & $h$ & 3.19 & $\mathrm{~mm}$ \\
Young's modulus & $E$ & $68.5 \times 10^{9}$ & $\mathrm{~Pa}$ \\
Mass density & $\rho$ & 2740 & $\mathrm{~kg} \mathrm{~m}^{-3}$ \\
Poisson ratio & $\nu$ & 0.33 & \\
Loss factor & $\eta$ & 0.004 & \\
\hline
\end{tabular}

1 4.2. System calibration

2 Before making a valid measurement, the electronic circuit used for both 3 controlling the actuator and detect the voltage and current at its input 4 terminals is subject to calibration. A known precision resistor is substituted for 5 the electrical load impedance to be measured and a scaling factor is calculated 6 for subsequent measurements. A picture of the homemade electronic circuit 7 used to perform the experiments is given in Fig. 8(b).

\subsection{Electrical input impedance}

9 Figure 9(a) illustrates the frequency response function of the actuator 10 electrical motional impedance when the base is prevented from vibrating, i.e. 11 for $v_{s}=0$ as given in Eq. (9). As can be seen in Fig. 9(a), there is a good 12 agreement between the measured and computed data. The actuator electrical 


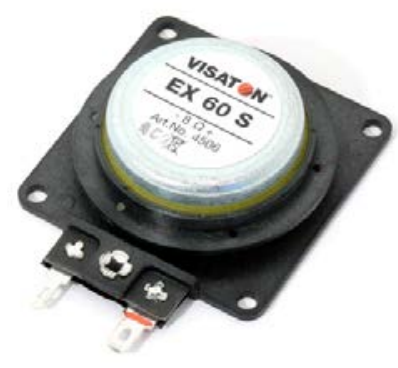

(a)

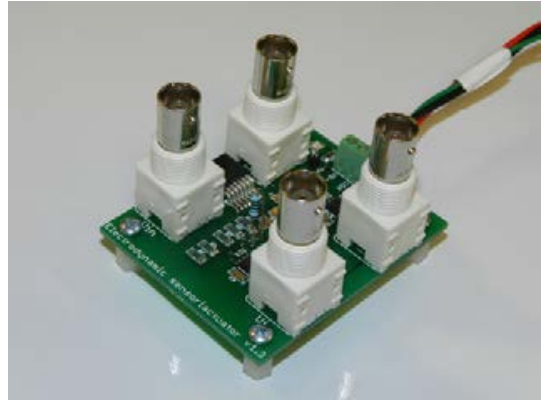

(b)

Figure 8: Pictures of the electrodynamic inertial actuator (a) and electronic circuit used for self-sensing actuation (b).

1 impedance is mainly resistive at low and middle frequencies, except around 2 the mechanical resonance at $75 \mathrm{~Hz}$ where there is also a large increase in the 3 actuator impedance, and becomes inductive as the frequency increases due to 4 the coil inductance.

${ }_{5}$ When attached to the plate, the vibration of the mass $M_{f}$ of the actuator 6 base depends on the plate mobility which in turns, will affect the actuator 7 electrical input impedance [37, as shown in Eq. (11). Figure 9(b) illustrates 8 the frequency response function of the input impedance of the actuator 9 when fixed near a corner of the plate (see Fig. 7(b)). As can be seen in 10 Fig. 9(b), there is a good agreement between the measured and computed 11 data. Compared to Fig. 9(a), it can be noted that the actuator resonance frequency, which can be seen at $75 \mathrm{~Hz}$ in Fig. 9(a), interferes with the first 13 structural resonance at $77 \mathrm{~Hz}$ for the plate used here. The following peaks in ${ }_{14}$ Fig. 9(b) are the natural frequencies of the plate which affect the actuator 15 electrical input impedance. 

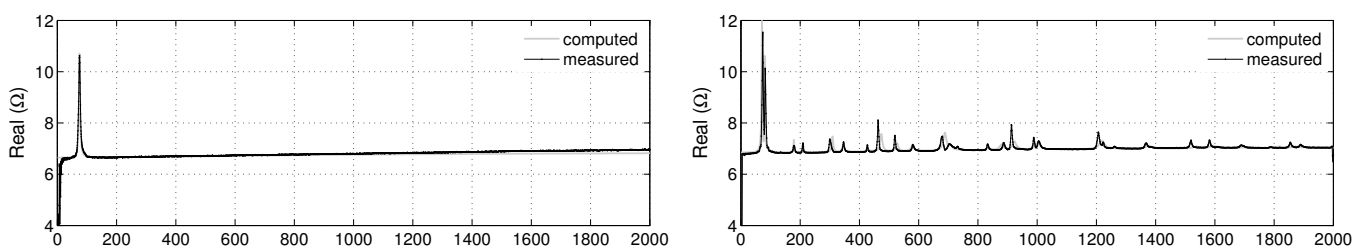

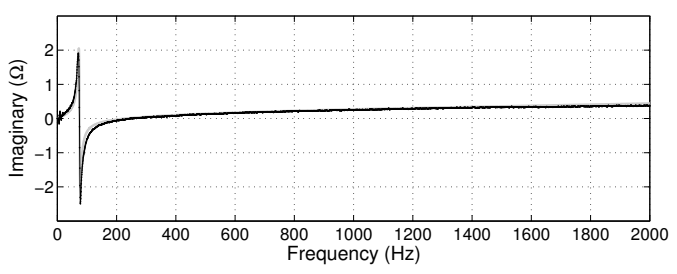

(a)

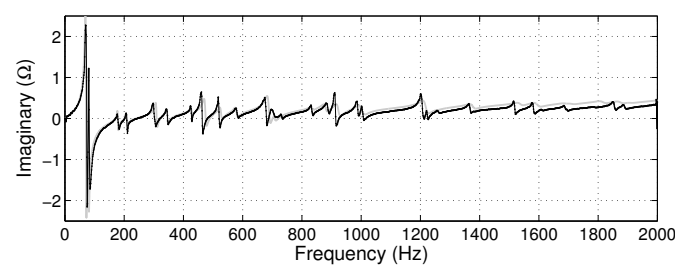

(b)

Figure 9: Computed and measured frequency response function of the actuator electrical input impedance when attached to a rigid foundation (a) and when mounted on the simply-supported plate (b).

\subsection{Structural response from variations in the actuator input impedance}

2 As given in Eqs. (12) and (13), the mobility function of the structure 3 under test can be derived from the actuator electromechanical impedances.

4 In order to investigate the validity and accuracy of the method and to define 5 the working range of the SMMD, we will first examine the measured FRF ${ }_{6} Z_{i n}(\omega)-Z_{i n 0}(\omega)$ shown in Fig. 10. As can be seen in Fig. 10 in the case of 7 the simply supported plate, there is a good agreement between simulation 8 and measurement in the frequency range from $60 \mathrm{~Hz}$ to $1000 \mathrm{~Hz}$. For higher 9 frequencies, the measured phase begins to deviate and crosses -90 degrees, in contradiction with the theory. This unwanted phase shift is presumably due to the non-ideal behavior of the voice coil transducer [39, 40, 41] from which the SMMD is developed. Therefore, the frequency range of the measured data presented below will be limited to $1 \mathrm{kHz}$. 

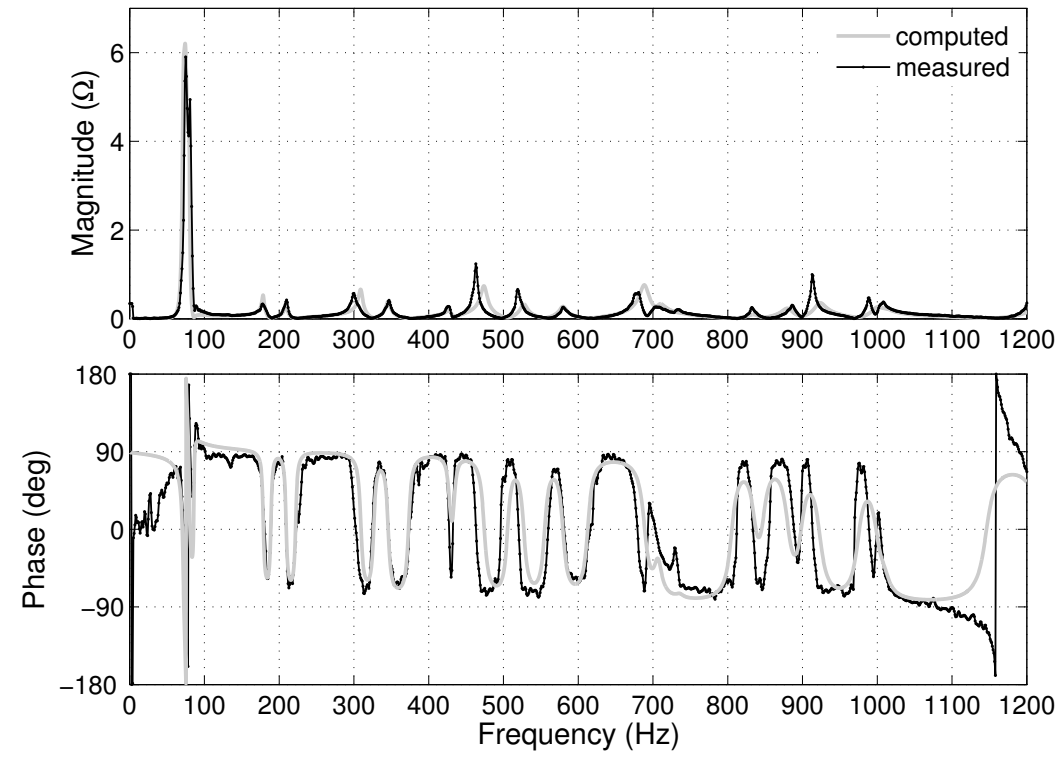

Figure 10: Computed and measured frequency response function $Z_{\text {in }}(\omega)-$ $Z_{i n 0}(\omega)$ showing the dynamic response of the structure from variations in the actuator electrical input impedance. 

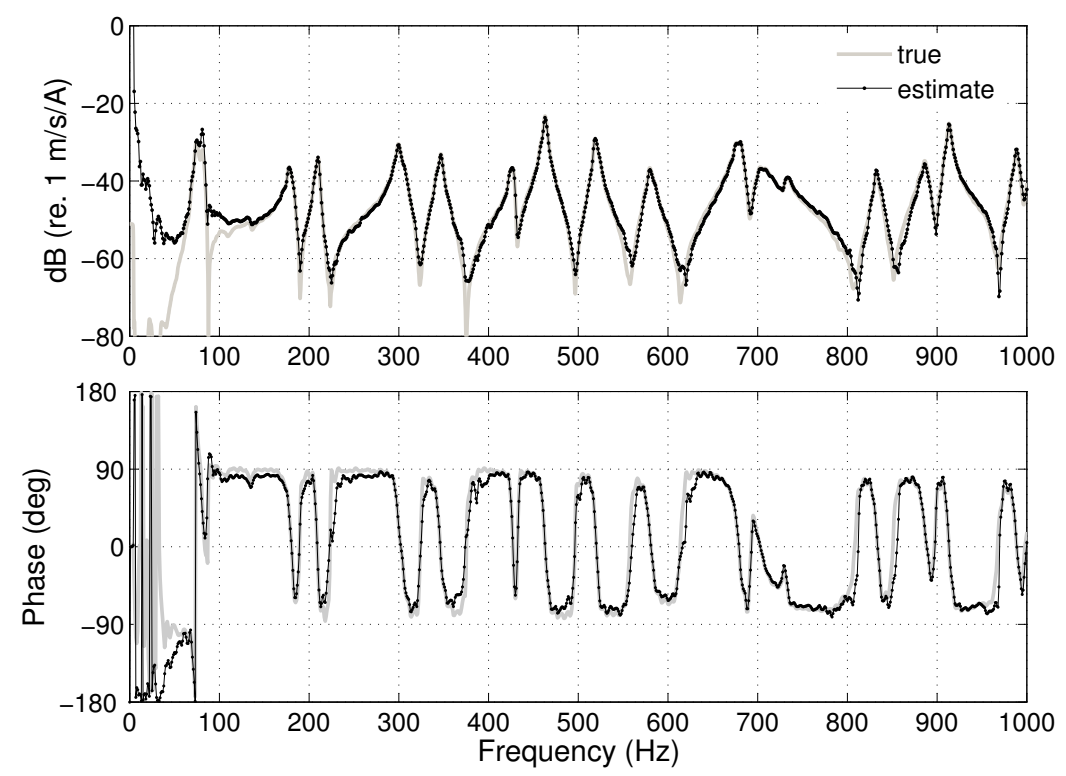

Figure 11: Velocity-current open loop frequency response function measured using a laser Doppler velocimeter (true) and derived from Eq. (12) (estimate).

1 4.5. Velocity-current open loop response

2 Figure 11 shows the comparison between the velocity-current open loop 3 frequency response function obtained using a laser Doppler velocimeter (LDV),

4 the beam of which is focused on the back side of the plate, and that derived 5 from Eq. (12). As can be seen in Fig. 11, there is a very good agreement 6 between measurement and estimate, showing the self-sensing capability of 7 the electrodynamic actuator. The deviation that can be observed around 75 $\mathrm{Hz}$ is due to the resonance of the actuator interfering with the first natural 9 resonance of the plate and thus influences the mobility measured using the 10 SMMD. 
1 4.6. Mobility function of the plate

2 Figure 12 illustrates the comparison between the mobility function of the 3 plate measured by shaker testing and that obtained from Eq. (13) using the 4 SMMD. As can be seen in Fig. 12, the two FRFs show a relatively good 5 agreement in the frequency range from $60 \mathrm{~Hz}$ to $1 \mathrm{kHz}$. The deviation that 6 can be seen above $700 \mathrm{~Hz}$ in Fig. 12 is most likely due to the SMMD location 7 on the plate which is not strictly the same as for shaker testing ; this effect is 8 moreover compounded by the fact that the SMMD is coupled to the structure 9 through a larger mounting base. As mentioned above, a deviation can also be

seen around the actuator resonance frequency which is very close to the first natural resonance of the plate used here. Notwithstanding the foregoing, Fig. 12 provides experimental evidence that the mobility function of a mechanical structure can be obtained using only an electrodynamic inertial exciter and without requiring external sensor.

\section{Discussion}

As confirmed experimentally in Section 4, the point mobility characteristics of a mechanical structure can be estimated with enough accuracy using the SMMD. With our mock-up SMMD prototype based on a commercially available electrodynamic inertial exciter, the measurement range already spans from $100 \mathrm{~Hz}$ to $1 \mathrm{kHz}$. Its working range is limited to lower frequencies due to the resonance of the actuator which interferes with the first natural resonance of the plate used here. An actuator with a lower resonance frequency should therefore improve the usable range of the SMMD. The limiting factors at higher frequencies are less evident, and may be related to the basic lumped-element 

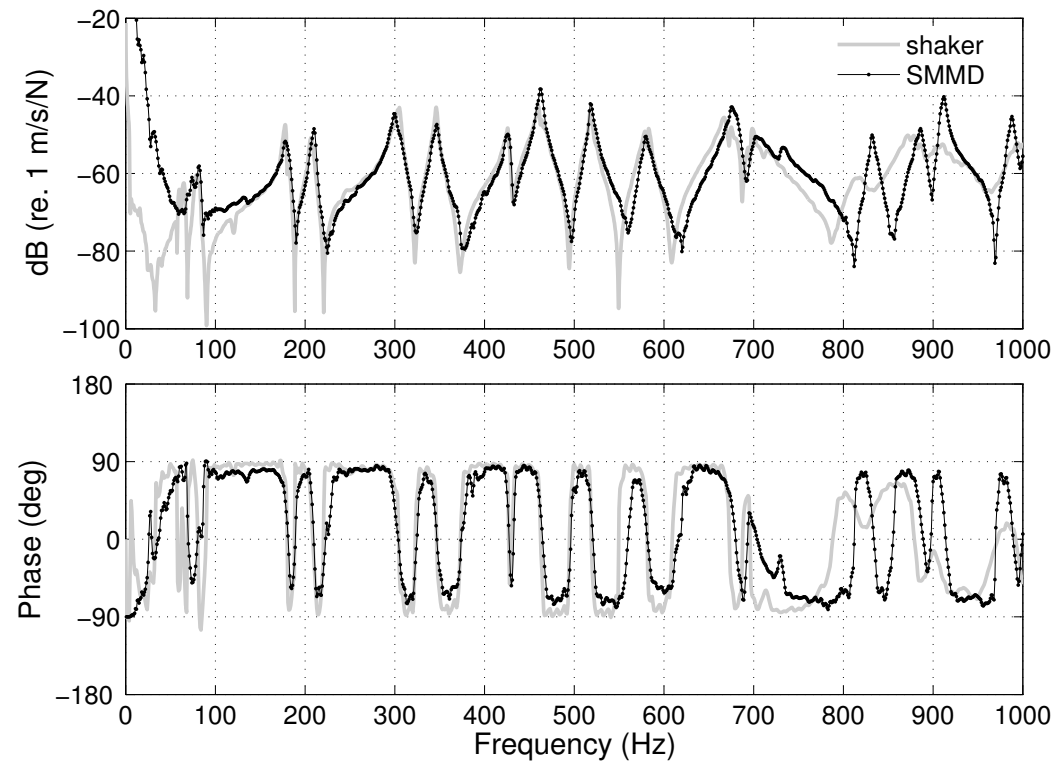

Figure 12: Comparison on the mobility function of the plate at the attachment point measured by shaker testing and derived from Eq. 13p (SMMD). 
model used here. As can be seen in Eqs. (12) 13), the derivation of the mobility function involves both the measured electrical impedances of the actuator and a few parameters identified experimentally for the model presented in Fig. 2 Discrepancies between these lumped models and the actual electrodynamic motors are described in the literature about loudspeakers and are likely to impact the SMMD as well. In particular, the non-ideal behavior of the voice coil transducer from which the SMMD is developed might be significant : it is recognized that eddy currents flowing in the iron pole structure may modify its electrical impedance [39, 40]. This is usually dealt with by employing lossy inductances in the model. A second possible cause is related to the suspension creep which is not covered by our simplified model [41]. An expanded model to incorporate eddy currents and suspension creep could then be considered in future work with a view to further extending the working range of a low-cost version the SMMD, or a dedicated (more expensive) device could be designed in order to reduce the above-mentioned effects.

The SMMD can also overcome some common issues encountered in practice in impact testing and shaker testing. Although impact testing is non-intrusive with a low mass-loading effect exerted upon the structure and therefore convenient for small lightweight structures, some practical problems related to the input force applied to the structure or the recording of the measurement signals may limit its use in some cases [37]. For example, multiple impacts, misalignment of impacts in both position and direction over the averaging period will affect the measured mobility characteristics of the structure under test. The short duration of the impact force may lead to overload the input channel of the multichannel analyzer, while supplying little energy into the 
structure. The duration of the contact time has also an effect on the frequency content of the force, a longer contact time leading to a reduced bandwidth. As a result, different force transducers have to be used according to the force needed [8] and impact hammers equipped with different masses may be required to cover the frequency range of interest. Note that impedance heads can also be used but these devices are not free of measurement error [5, 14. On the other hand, shaker testing can supply more energy to the structure over a longer period of time and hence provide better control of the frequency content, amplitude and duration of the mechanical excitation. A grounded mounting support is necessary, however, which can make it difficult to fit into small spaces. In addition, shaker testing can significantly influence the dynamic characteristics of the structure under test. The stinger has to be axially stiff but soft in bending in order to reduce the transmission of bending moments to the armature of the shaker on one hand and the force transducer and structure under test on the other hand [37]. With the SMMD, no direct measurement of the applied force with a force transducer is necessary. As the force excitation and velocity response occur through the electrodynamic transducer, there is no misalignment of sensors. The applied force and resulting velocity are therefore colocated and constrained to a single direction of action by the voice coil transducer, allowing highly repeatable measurement. Various electrodynamic inertial exciters are commercially available that can suit a wide range of dynamic structures. In addition, the mass-loading effect exerted upon the structure by the actuator can also be removed mathematically, allowing measurement of the unloaded mobility characteristics as described in [15]. However, one drawback of such a mobility 
measuring device based on an electrodynamic inertial exciter is that it is coupled to the structure through a relatively large mounting base, which can hardly be considered as a point.

Compared to the concept proposed by Sands et al. [15] which is also based on a voice coil actuator, the SMMD is developed from an "off-the-shelf" inertial exciter and does not require extra sensor to determine the mobility function of the structure under test. Using a SMMD, strong electric signals lead to a very good Signal-to-Noise Ratio and the mobility function of the structure can be evaluated by a single measurement for a broad frequency range, typically from $100 \mathrm{~Hz}$ to $1 \mathrm{kHz}$ with the current SMMD prototype, using a sweep or random test signal over a duration of about one minute. In comparison to the experimental technique proposed by Ling et al. (2001) [26] for detecting mechanical impedance of structures using the sensing capabilities of a piezoceramic inertial actuator, no force sensor is required to measure the parameters of the transduction matrix. Significant discrepancies are reported in [26] at the anti-nodes of the beam structure in their frequency range of interest. According to the authors, it arises from the non-ideal linearity between the electrical variables and mechanical variables due to the construction of the piezoelectric inertial actuator. Above the actuator resonance frequency, the dynamic range of the SMMD allows the anti-nodes and nodes to be well identified from the derived point mobility function.

The mobility characteristics of a structure obtained using a SMMD can also be informative in other contexts. Since the actuator electrical input impedance is a sensitive function of the mechanical impedance of the structure at the attachment point, variations thereof may be used to detect changes 
of structural properties such as stiffness changes due to mechanical failure, mass changes due to wearing, or changes of supporting conditions due to malfunction of a coupled mechanical element [7, 27, 28, 29]. In addition to determining the point mobility of a structure, the SMMD can also be used as a self-sensing actuator [10] to achieve active vibration control of the structure without having to remove it. This may help predicting the maximum feedback gain that can be implemented for a specific application, and hence the stability of the feedback loop where the control unit is mounted [12].

\section{Conclusion}

This paper presents a stand-alone experimental tool for measuring the mobility characteristics of a mechanical structure using only an electrodynamic inertial exciter. Unlike the state of the art techniques such as impact testing and shaker testing, it is shown that mobility measurement can be performed with good accuracy without involving external sensors such as accelerometers, load cells or impedance heads, but directly from variations in the electrical input impedance of the actuator fixed onto it. The electrical input impedance is available when switching on the device or can be assessed in operation, allowing early detection of structural failure, defects or other changes. The proof of concept is demonstrated using a commercially available actuator relatively inexpensive, leading to a good dynamic range from $100 \mathrm{~Hz}$ to $1 \mathrm{kHz}$. It is believed that the formulations presented in this paper can be used to investigate new designs of electrodynamic actuator in order to optimize the performance properties of this type of self-sensing actuator depending on the structure to be characterized. 


\section{Acknowledgment}

2 This work was supported by the Swiss National Science Foundation (SNSF)

3 under grant PBELP2-146567, the National Sciences and Engineering Research 4 of Canada (NSERC) and Bombardier Aerospace.

\section{Appendix A. Derivation of the sensoriactuator transfer functions}

6 From Eqs. (1), the reaction force at the base of the actuator can be 7 written as

$$
f_{s}=\frac{j \omega M_{a}}{Z_{m a}(\omega)} B l i-\left(j \omega M_{a} \frac{Z_{m s}(\omega)}{Z_{m a}(\omega)}+\mathrm{j} \omega M_{f}\right) v_{s}
$$

8 where $Z_{m a}(\omega)=Z_{m s}(\omega)+\mathrm{j} \omega M_{a}$, and the velocity of the proof mass $M_{a}$ may 9 be expressed as

$$
v_{a}=-\frac{B l}{Z_{m a}(\omega)} i+\frac{Z_{m s}(\omega)}{Z_{m a}(\omega)} v_{s}
$$

10 Substituting the mobility function given by Eq. (8) into Eq. (A.1), the transfer function between the input current and the reaction force at the attachment point can therefore be written as

$$
H_{i}(\omega)=\frac{f_{s}}{i}=\frac{\mathrm{j} \omega M_{a} B l}{Z_{m a}(\omega)+\mathrm{j} \omega M_{a}\left(Z_{m s}(\omega)+\frac{M_{f}}{M_{a}} Z_{m a}(\omega)\right) Y(\omega)}=\left[T_{21}(\omega)+T_{22}(\omega) Y(\omega)\right]^{-1}
$$

13 Note that Eq. A.3 is equivalent to Eq. (10).

15 Considering now that the proof mass $M_{a}$ is moving as a result of an external force acting on the actuator base, i.e. for $i=0$, the complex ratio

17 between the output and the input velocities can be written as [33, 34]

$$
T_{a}(\omega)=\left.\frac{v_{a}}{v_{s}}\right|_{i=0}=\frac{Z_{m s}(\omega)}{Z_{m s}(\omega)+\mathrm{j} \omega M_{a}}
$$


1 which denotes the velocity transmissibility between the proof mass and the 2 actuator base.

3

4 Substituting Eq. A.2 into Eq. (1), the output voltage when the actuator 5 is driven by a current source is

$$
u=\left(Z_{e}(\omega)+\frac{(B l)^{2}}{Z_{m a}(\omega)}\right) i+B l\left(1-\frac{Z_{m s}(\omega)}{Z_{m a}(\omega)}\right) v_{s}
$$

15

16

and after some further manipulation, the output velocity at the actuator base can be rewritten in terms of the input current and the induced voltage at the transducer terminals, as

$$
v_{s}=\frac{Z_{m a}(\omega)}{\mathrm{j} \omega M_{a} B l}\left(u-\left(Z_{e}(\omega)+\frac{(B l)^{2}}{Z_{m a}(\omega)}\right) i\right)
$$

When attached to a rigid foundation, i.e. for $v_{s}=0$, the actuator electrical input impedance (in $\Omega$ ) can be derived from Eq. A.5 as

$$
Z_{i n 0}(\omega)=\left.\frac{u}{i}\right|_{v_{s}=0}=Z_{e}(\omega)+\frac{(B l)^{2}}{Z_{m a}(\omega)}=\frac{T_{11}(\omega)}{T_{21}(\omega)}
$$

and substituting now Eq. (8) into Eqs. (1), the electrical input impedance of the actuator when attached to the structure can therefore be expressed as

$$
Z_{i n}(\omega)=\frac{u}{i}=Z_{e}(\omega)+\frac{(B l)^{2}}{Z_{m s}(\omega)+\mathrm{j} \omega M_{a} \frac{1+\mathrm{j} \omega M_{f} Y(\omega)}{1+\mathrm{j} \omega\left(M_{a}+M_{f}\right) Y(\omega)}}=\frac{T_{11}(\omega)+T_{12}(\omega) Y(\omega)}{T_{21}(\omega)+T_{22}(\omega) Y(\omega)}
$$

3 Note that Eqs. (A.7) and (A.8) are equivalent to Eq. (9) and (11), respectively.

15

The transfer function between the input current and the output velocity at the attachment point can be derived from Eqs. A.6. A.8) as

$$
G_{i}(\omega)=\frac{v_{s}}{i}=\frac{Z_{m a}(\omega)}{j \omega M_{a} B l}\left(Z_{i n}(\omega)-Z_{i n 0}(\omega)\right)=T_{21}(\omega)\left(Z_{i n}(\omega)-Z_{i n 0}(\omega)\right)
$$


1 Note that Eq. A.9 is equivalent to Eq. (12). From Eqs. (A.3) and (A.9),

2 the mobility function of the structure at the attachment point can also be

3 expressed as

$$
Y(\omega)=\frac{v_{s}}{f_{s}}=\frac{G_{i}(\omega)}{H_{i}(\omega)}
$$

4 Substituting Eqs. A.3 and (A.9) into Eq. A.10 yields

$$
Y(\omega)=G_{i}(\omega) \frac{Z_{m a}(\omega)}{j \omega M_{a} B l}+\frac{G_{i}(\omega)}{B l}\left(Z_{m s}(\omega)+\frac{M_{f}}{M_{a}} Z_{m a}(\omega)\right) Y(\omega)
$$

5 and after some further manipulations, Eq. A.11 can be expressed as a 6 function of Eq. (A.9) as

$$
Y(\omega)=\frac{Z_{m a}(\omega)}{\mathrm{j} \omega M_{a} B l} \frac{G_{i}(\omega)}{1-\frac{1}{B l}\left(Z_{m s}(\omega)+\frac{M_{f}}{M_{a}} Z_{m a}(\omega)\right) G_{i}(\omega)}=\frac{T_{21}(\omega) G_{i}(\omega)}{1-T_{22}(\omega) G_{i}(\omega)}
$$

7 Note that Eq. A.12 is equivalent to Eq. 13.

\section{Appendix B. Derivation of the electromechanical parameters}

9 The proof mass $M_{a}$ is determined in two steps by measuring the velocity transmissibility of the actuator when attached to the shaker table at its base

11 (see Fig. 6(a)), and then combined with an added mass $m$ (see Fig. 6(b)). and without added mass, respectively, the proof mass $M_{a}$ can be derived after

14

$$
M_{a}=\frac{f_{m}^{2}}{f_{n}^{2}-f_{m}^{2}} m
$$

and the effective stiffness $K_{a}$ of the suspension can therefore be derived as

$$
K_{a}=\left(2 \pi f_{n}\right)^{2} M_{a}
$$


Similarly, the mass $M_{f}$ of the actuator base can be determined from the 2 transmissibility response as

$$
M_{f}=\frac{K_{a}}{\left(2 \pi f_{f}\right)^{2}}
$$

3 where $f_{f}$ is the resonance frequency when the actuator is mounted upside 4 down on the shaker (Fig. 6(c)).

5 The effective mechanical resistance $R_{a}$ can be derived from the peak6 transmissibility which is the maximum amplitude of the transmitted velocity 7 for a given amplitude of the input velocity. For a linear system the peak8 transmissibility is $\left|T_{a}(\omega)\right|_{\max } \simeq 1 /(2 \zeta)$, where $\zeta=R_{a} /\left(2 \sqrt{K_{a} M_{a}}\right)$ is the 9 damping ratio (see Eq. A.4), and the effective mechanical resistance is therefore given by

$$
R_{a}=2 \zeta \sqrt{M_{a} K_{a}}
$$

11 Once $R_{a}$ is known, the electrodynamic transduction coefficient $B l$ can be 12 derived from the actuator electrical motional impedance as

$$
B l=\sqrt{R_{a}\left(R_{e m}-R_{e}\right)}
$$

13

where $R_{e}$ is the dc resistance of the coil and $R_{e m}$ is the electrical motional resistance measured at the actuator resonance frequency $f_{n}$.

\section{References}

[1] A. G. Webster, Acoustical impedance and theory of horns and of the phonograph, Proceedings of the National Academy of Sciences of the United States of America 5 (7), Washington, 1919, pp. 275-282. 
1 [2] F. A. Firestone, A new analogy between mechanical and electrical systems, $2 \quad$ The Journal of the Acoustical Society of America 4 (1933) 249-267.

3 [3] G. J. O'Hara, Mechanical impedance and mobility concept, The Journal $4 \quad$ of the Acoustical Society of America 41 (1966) 1180-1184.

5 [4] P. Gardonio and M. J. Brennan, On the origins and development of 6 mobility and impedance methods in structural dynamics, Journal of $7 \quad$ Sound and Vibration 249 (2002) 557-573.

8 [5] D.J. Ewins, Modal Testing: Theory and Practice, Research Studies Press $9 \quad$ LTD, Letchworth, Hertfordshire, England, 1984.

[6] H. P. Olesen, Measurement of the dynamic properties of materials and structures, Brüel and Kjaer Application Note No. 17-180, 1977.

[7] P. Cawley, The sensitivity of the mechanical impedance method of nondestructive testing, NDT International 20 (4) (1987) 209-215.

[8] H. P. Olesen and R. B. Randall, A guide to mechanical impedance and structural response techniques, Brüel and Kjaer Application Note No. 17-179, 1977.

[9] R. Boulandet, A. Pelletier, P. Micheau and A. Berry, Active vibration control using self-sensing actuators: an experimental comparison of piezoelectric and electromagnetic technologies, Proceedings of the ASME 2014 International Mechanical Engin. Congress 83 Exposition (IMECE), Montreal, Canada, 2014. 
1 [10] R. Boulandet, M. Michau, P. Micheau and A. Berry, Aircraft panel 2 with active sound power reduction capability through virtual mechanical $3 \quad$ impedances, Journal of Sound and Vibration 361 (2016) 2-19.

4 [11] S.J. Elliott, L. Benassi, M.J. Brennan, P. Gardonio and X. Huang, Mo5 bility analysis of active isolation systems, Journal of Sound and Vibration $6 \quad 271(1-2)(2004) 297-321$.

7 [12] J. Rohlfing, P. Gardonio and S. J. Elliott, Base impedance of velocity 8 feedback control units with proof-mass electrodynamic actuators, Journal $9 \quad$ of Sound and Vibration 330 (20) (2011) 4661-4675.

[13] W. G. Halvorsen and D. L. Brown, Impulse technique for structural frequency response testing, Sound and Vibration (1977) 8-21.

[14] J.M.W. Brownjohn, G.H. Steele, P. Cawley and R.D. Adams, Errors in mechanical impedance data obtained with impedance heads, Journal of Sound and Vibration 73 (3) (1980) 461-468.

[15] S.A. Sands and P. Camilleri and R.A. Breuer and G.K. Campbell and R.J. Alfredson and E.M. Skuza and P.J. Berger and M.H. Wilkinson, A novel method for the measurement of mechanical mobility, Journal of Sound and Vibration 320 (3) (2009) 559-575.

[16] N. W. Hagood and E. H. Anderson Simultaneous sensing and actuation using piezoelectric materials, Proceedings of the SPIE 1543 Active and adaptive optical components, San Diego, CA, 1991. 
1 [17] J. J. Dosch, D. J. Inman and E. Garcia, Self-sensing piezoelectric actu2 ator for collocated control, Journal of Intelligent Material Systems and $3 \quad$ Structures 3 (1992) 166-185.

4 [18] J. S. Vipperman and R. L. Clark, Implementation of an adaptive piezoelec5 tric sensoriactuator, American Institute of Aeronautics and Astronautics, $6 \quad$ Vol. 34, No. 10, (1996) 2102-2109.

7 [19] J. S. Vipperman and R. L. Clark, Multivariable feedback active structural $8 \quad$ acoustic control using adaptive piezoelectric sensoriactuators, The Journal $9 \quad$ of the Acoustical Society of America 105 (1999) 219-225.

[20] S. O. R. Moheimani, A survey of recent innovations in vibration damping and control using shunted piezoelectric transducers IEEE Transactions on Control Systems Technology 11 (2003) 482-494.

[21] F. Hong and C.K. Pang, Robust vibration control at critical resonant modes using indirect-driven self-sensing actuation in mechatronic systems, ISA Transactions 51 (2012) 834-840.

[22] Ch. Paulitsch, P. Gardonio and S. J. Elliott, Active vibration control using an inertial actuator with internal damping, The Journal of the Acoustical Society of America 119 (2006) 2131-2140.

[23] D.J. Leo and D. Limpert, A self-sensing technique for active acoustic attenuation, Journal of Sound and Vibration 235 (5) (2000) 863-873.

[24] B. Hanson and M. Levesley, Self-sensing applications for electromagnetic actuators, Smart Materials Structures 5 (1996) 345-351. 
1 [25] C. Liang, F. Sun, and C.A. Rogers, Electro-mechanical impedance mod$2 \quad$ eling of active material systems Sensors and Actuators A: Physical 5 (2) 3 (2001) 171-186.

4 [26] S-F. Ling and Y. Xie, Detecting mechanical impedance of structures $5 \quad$ using the sensing capability of a piezoceramic inertial actuator Sensors $6 \quad$ and Actuators A: Physical 93 (3) (2001) 243-249.

7 [27] S-F. Ling and Y. Xie, Monitoring structural integrity using a piezoelectric

[28] O. Doutres, N. Dauchez, J-M. Genevaux, G. Lemarquand, and S. Mezil, Ironless transducer for measuring the mechanical properties of porous materials Review of Scientific Instrument 812010 055101-8.

[29] C. Bois, Ph. Herzog and C. Hochard, Delamination characterization in laminated composites using in-situ measurements and parametric identification Journal of Sound and Vibration 299 (4-5) (2007) 786805.

[30] W. Klippel, Nonlinear large-signal behavior of electrodynamic loudspeakers at low frequencies Journal of the Audio Engineering Society 40 (1992) 483-496.

[31] F. V. Hunt, Electroacoustics: the analysis of transduction and its historical background, American Institute of Physics for the Acoustical Society of America, 2nd edition, 1982.

[32] F. Fahy and P. Gardonio, Sound and structural vibration, 2nd edition, Academic Press, Oxford, UK, 2007 
1 [33] S. S. Rao, Mechanical Vibrations, 2nd Edition, Addison-Wesley, New $2 \quad$ York, USA, 1990.

3 [34] S. J. Elliott, M. Serrand, P. Gardonio, Feedback stability limits for active $4 \quad$ isolation systems with reactive inertial actuators Journal of Vibration and $5 \quad$ Acoustics 123 (2) (2001) 250-261.

6 [35] R. H. Small, Direct radiator loudspeaker system analysis Journal of the

7

8 Audio Engineering Society 20 (1972) 383-395.

[36] O. Robin, J-D. Chazot, R. Boulandet, M. Michau, A. Berry, N. Atalla, A Plane and Thin Panel with Representative Simply Supported Boundary Conditions for Vibroacoustic Tests Acta Acustica united With Acustica 102 (2016) 170-182.

[37] K. G. McConnel, Vibration testing : Theory and practice, John Wiley 83 Sons, INC, New York, USA, 1995.

[38] C. R. Fuller, S. J. Elliott and P. A. Nelson, Active control of vibration, Academic Press Inc., San Diego, USA, 1996.

[39] J. Vanderkooy, A model of loudspeaker driver impedance incorporating Eddy current in the pole structure Journal of the Audio Engineering Society 37 (1989) 119-128.

[40] W. M. Leach, Loudspeaker voice-coil inductance losses : circuit models, parameter estimation and effect on frequency response Journal of the Audio Engineering Society 50 (2002) 442-449. 
1 [41] M. H. Knudsen and J. Grue Jensen, Low-frequency loudspeaker models 2 that include suspension creep Journal of the Audio Engineering Society $3 \quad 41(1992) 3-18$. 